\title{
Peer Pasternack
}

\section{Osthochschulen}

\section{Eine Wegbeschreibung mit kurzweiligen Abschweifungen}

Vor bald sieben Jahren kündigte der ostdeutsche Kleinbürger den mit seinen kleinbürgerlichen Anführern geschlossenen Gesellschaftsvertrag Versorgung gegen Anpassung auf. Seitens der Herrschaftsunterworfenen war das durchaus mit heroischen Anwandlungen verbunden. Deshalb nennen wir dies seither die erste gelungene Revolution in Deutschland. Die Akademiker waren nicht deren Speerspitze gewesen. Das einte sie mit Gemüsehändlern, Produktionsarbeitern oder Betriebsabteilungsleitern. Es gab keine sozialmilieubestimmte Gruppe, die Träger des überraschenden Vorgangs war. Freilich gab es Einzelne aus all den Gruppen, die als Impulsgeber und Aktivisten wirkten. Näherhin gab es dann auch größere Gruppen, die die emanzipatorischen Chancen des Aufbruchs zu nutzen suchten, etwa anfänglich die Studierenden. Nur weil der ostdeutsche Kleinbürger sich an den Abläufen an prominenter Stelle beteiligt hatte, konnten sie etwas bewirken, und genau deshalb, weil er sich beteiligt hatte, mündete das Ganze schließlich in ein gescheitertes Gelingen.

Die Demystifizierung des Ausgangspunktes ist die eine Voraussetzung zur Erklärung der unterdessen im Osten entstandenen Einheitsfront der Großen Klage, die auch die Hochschulen erfaßt hat. Die andere Voraussetzung ist eine Herausarbeitung dessen, was den vom Ressentiment entkleideten rationalen Kern der Klage bildet. Für die Hochschulen ist das so besonders notwendig wie schwierig: Kein anderer Bereich immerhin, der bereits 1989 nicht allein in Ost-, sondern auch in Westdeutschland als derart dringend reparaturbedürftig galt. Kein anderer zugleich, der heute so schwierig durchschaubar ist. Zonale Mentalitäten verbergen sich hinter g'schwind akkulturiertem westlichen Habitus. Letzterer ist wochentäglichen Gästen abgeschaut, die vielfach die Rolle des Entscheiders geben, um zum regelmäßig verlängerten Wochenende dort zu siedeln, wo sie herkamen.

Zunächst ist ja in der Tat ein Zusammenprall zweier deutlich verschiedener Wissenschaftssysteme und -kulturen zu bewältigen. Auf der einen Seite die vom angelsächsischen Einfluß nicht gänzlich unberührte westdeutsche Beamtenwissenschaft mit ihrem der Pampersindustrie entlehnten Produkt- 
marketing. Auf der anderen die zuvor an den herausragenden Leistungen der Sowjetwissenschaft orientierte zentralplangesteuerte Erkenntnissuche im Dienste der Erfüllung der Hauptaufgabe der Realisierung der Einheit von Wirtschafts- und Sozialpolitik. Dieser nicht zu vermeidende Zusammenprall verführte anfangs manchen auch im Westen zu einer euphorisierenden Idee: Die ostdeutsche Hochschulerneuerung könnte doch zum Ausgangspunkt einer gesamtdeutschen Hochschulreform werden. Das hätte sie vielleicht (wobei damit noch nichts über die wahrscheinliche Richtung dieser imaginierten Reform gesagt ist), wenn gelegentlich eine Frage ernsthaft $\mathrm{zu}$ beantworten versucht worden wäre: Warum eigentlich müssen zwei unterschiedliche Wissenschaftssysteme um jeden Preis in kürzester Zeit vereinheitlicht werden?

Drei Antworten sind gegeben worden. Erstens, weil wir das in allen Bereichen so machen, denn es ist der Wählerwille: Eine Aussage von hochnormativer Begründungskraft. Zweitens, weil wir Chancengleichheit für die studierenden und lehrenden Brüder und Schwestern herstellen müssen: Ein Zeugnis eindrucksvollen Selbstbewußtseins, gestützt durch die nicht eindruckslosen Zustände des westdeutschen Hochschulsystems. Und drittens, weil alles andere viel zu teuer wäre: Das hilft uns, die Mechanismen von Verteilungspolitik zu begreifen. Denn der Massenabbau akademischer Beschäftigungsmöglichkeiten etwa ist, bruttosozialproduktiv betrachtet, selbstredend kostenneutral.'

Damit sehen wir bei den Ost-Hochschulen bereits, was auch über sie hinaus gilt: Ursachen für Änderungen oder Nichtänderungen an Hochschulen sind höchstens zum Teil in diesen selbst zu suchen. Der spezifische Charakter einer Arbeit, die Wissenschaft und Bildung zusammenführt, hatte einst zu der Auffassung geführt, daß diese Tätigkeit besser unmittelbarer Verwertungslogik entzogen sein sollte. Die Unterhaltung der Hochschulen fand sich zum Bestandteil öffentlicher Daseinsvorsorge geadelt. Die öffentliche Finanzierung der Einrichtungen und die relative Freiheit ihres Tuns von unmittelbaren Nützlichkeitserwägungen bringt zugleich eine extrem asymmetrische Verteilung der Sanktionsgewalt mit sich. Wo der

1 Ende 1993 bereits war die Zahl der beschäftigten ostdeutschen WissenschaftlerInnen auf $30 \%$ des 1990er Ausgangswertes reduziert (Meske 1993, 29). Eine allein auf die Hochschulen bezogene Betrachtung ergibt immerhin noch eine Reduzierung der Personalstellen auf 54\% bis zum Jahresende 1994 (Wissenschaftsrat 1996, 164). Zur Einordnung dieser Zahlen ist es sinnvoll, vergleichend Berechnungen der Forschungsgruppe Wissenschaftsstatistik am WZB heranzuziehen: Sie gibt an, die DDR-Wissenschaftsstatistiken nach OECD-Kriterien umgerechnet und damit Vergleichbarkeit mit der bundesdeutschen Statistik hergestellt zu haben. Ergebnis dessen sei u.a. die Feststellung, daß die DDR 1987 je 1.000 Erwerbstätige 16,3 in Wissenschaft/Forschung/Entwicklung (also in Hochschul-, Akademie-, Industrie- und Ressortforschung) Beschäftigte gehabt habe, die Bundesrepublik 15,6. Diese Differenz sei von zu vernachlässigender Größenordnung (Melis 1993, 352). 
staatliche Geldgeber geben oder vorenthalten kann, da können die Hochschulen nur nehmen: Gleich was und wieviel, sie müssen akzeptieren. Die Palette ihrer möglichen Sanktionsinstrumente ist recht beschränkt. Es kann eine - seit geraumer Zeit eher gleichgültige - Öffentlichkeit zu interessieren versucht werden. Als Bestandteil von Protestverhalten können gezielte Regelüberschreitungen eingesetzt werden, um anzuzeigen, daß nun aber wirklich das Maß voll sei. Arbeitsverweigerungen von WissenschaftlerInnen und Studierenden gefährdeten den gesellschaftlichen Reproduktionszusammenhang erst dann, wenn sie jahrelang durchgehalten würden. Da das niemand tut, rufen sie beim Publikum regelmäßig eher verständnisloses Kopfschütteln hervor. Die zwangsläufige Folge sind Ritualisierungen der (bspw. studentischen) Protestkultur mit entsprechendem Vernutzungseffekt.

Diese Mechanismen asymmetrischer Verteilung der Sanktionsmittel lemten die ostdeutschen Hochschulen recht zeitig kennen. Ein an sie häufig adressierter Vorwurf lautete, sie hätten anfangs trotz genügender Zeit keine zureichenden Anstrengungen zur Selbsterneuerung unternommen. (Dabei wäre freilich gerechterweise die schon angedeutete Frage zu behandeln: Inwieweit haben Hochschulen überhaupt und namentlich deutsche je zureichende Eigenreform-Kapazitäten auszubilden vermocht?) Für die insuffiziente Selbsterneuerung gab es Gründe. So konnte der Vorgang nach Lage der Dinge, zuvörderst der politischen Erwartungen, keine soziale, berufliche, akademische Perspektive für die potentiellen Erneuerer garantieren. Die Wissenschaftler - das wurde schneller klar als in anderen Bereichen, auch wenn es sich hier großteils erst später als dort realisiert fand - hatten mehr zu verlieren als ihre Ketten. Nicht allein der Gewinn wissenschaftlicher Freiheit stand ins Haus. Es drohte auch, zumindest für einen Großteil, der Verlust des Arbeitsplatzes: aufgrund von tatsächlichen und aufgrund von vermeintlichen politischen Belastungen, wegen negativer fachlicher Evaluierung wie infolge des absehbaren Personalabbaus, bedingt durch die (zunächst antizipierten und hernach auch so oder deutlicher getroffenen) haushaltspolitischen Entscheidungen.

Dies resultierte dann in eine spezifische Form der allerorten im ostdeutschen Siedlungsgebiet beobachtbaren Verschränkung von sozialen Abstiegsprozessen mit sozialen Aufstiegsprozessen. ${ }^{2}$ Die mangelnde Berechenbarkeit der Prozesse durch ungeklärte oder unklare Bewertungs- und Entscheidungsmaßstäbe war dabei keine sonderlich geeignete Grundlage

2 In den historisch-hermeneutisch orientierten Geisteswissenschaften etwa sind, wie eine soeben abgeschlossene Untersuchung ergeben hat, 61\% der 1990 tätig gewesenen OstProfessorInnen 1995 nicht mehr als solche tätig und zugleich 13,5 derjenigen wissenschaftlichen MitarbeiterInnen, die trotz Strukturwandel in akademischer Beschäftigung verblieben, in den Professorenstatus aufgestiegen (Pasternack 1996, 159). 
für massenhaftes Engagement, gar nonkonformistisches oder konfliktorientiertes Verhalten in diese oder jene Richtung. ${ }^{3}$

Damit kam die Stunde der externen Akteure. Das waren Ministerien, bundesdeutsche Wissenschaftsgremien und westdeutsche Professoren als Gründungsbeauftragte, Evaluatoren und dgl. Sie erachteten eingangs zweierlei als nötig. Einesteils war die Nachhaltigkeit der Verunsicherung des vorhandenen Hochschulpersonals zu inszenieren. Damit konnte der Gefahr von Solidarisierungen, mithin der Organisierung von Interessen weitgehend begegnet werden. Andernteils wurde es - eine pragmatische organisationstechnische Entscheidung - als notwendig angesehen, einen kompletten Elitenwechsel im ostdeutschen Hochschulwesen zu vermeiden. Also fand sich die erste Reihe der wissenschaftlichen Funktionselite ausgewechselt, und die zweite Reihe rückte nach. Deren wesentliche Qualifikation bestand, bis auf wenige Ausnahmen, darin, in der DDR unauffällig gewesen zu sein: mithin einen wesentlichen Beitrag zur Stabilisierung der bornierten Verhältnisse geleistet zu haben. Das in diesem Zusammenhang betriebene atemlose Sortieren des Personals nach belastet und unbelastet verschaffte den hektisch damit Befaßten offensichtlich gezielt gesetzte Blackouts. Bei der Exzessivität des Schlachtfestes konnte eines freilich nicht ausbleiben: Mitunter erwischte es auch die Richtigen.

Die Verunsicherung des Personals und der selektive Charakter der Personalüberprüfungen sicherten vornehmlich eines: Die kleinbürgerlich-pragmatischen Dispositionen - die wesentlich die längere Existenz der DDR, so wie sie war, ermöglicht hatten - und die staatsfixierte Sichtweise der vormals 'kommunistisch' dominierten Professorenschaft gingen dem Personal auch in den neuen Strukturen nicht verloren (wenn wir einmal annehmen wollen, daß da vielleicht Gefahren bestanden hätten). Im akademischen Milieu Ostdeutschlands zeigte sich diese Kleinbürgerlichkeit vornehmlich in einer Wollustorgie des Opportunismus der übergroßen Mehrheit. (Von Westdeutschland sollten wir ein andernmal reden.) Man war interessiert am eigenen Durchkommen und im übrigen eher gelangweilt von den sonst verhandelten Dingen. Für das Durchkommen galt: Das beste Mittel, den diversen Abwicklungen zu entgehen, ist, irgendwie bei denjenigen zu sein, die sie anordnen. Die wenigen bereits in der DDR konfliktfähigen WissenschaftlerInnen finden sich zwischenzeitlich meist nicht mehr an den Hochschulen. Sie können folglich unseren Befund nicht trüben.

Anfangs freilich wollten tatsächlich kleine aktive Minderheiten an den OstHochschulen den großen Angriff starten. Das ordinarial inspirierte Hochschulrecht der ehemaligen Bundesrepublik sollte aus den Angeln gehoben werden. Es war so schön, grundsätzlich zu sein. Die altlastgesättigte Pro-

3 Genauer dazu Brentjes/Pasternack (1994) und Pasternack (1995). 
fessorenschaft fand sich unter gezielter Ausnutzung ihres schlechten Gewissens zu demokratischen Verfahren gedrängt. Konzile wurden in Viertelparitäten gewählt. Die neuen Uni-Verfassungen schrieben plebiszitäre Fingerübungen fest. Hochschulen als öffentliche Orte sollten entstehen, die vor allem eines aus der Vergangenheit gelernt haben: Daß Staatsferne, Kritikfähigkeit und interne demokratische Verfaßtheit die Minimalvoraussetzungen sind, um Freiheit von Forschung, Lehre und Studium zu sichern und ein Faktor im öffentlichen Diskurs zu sein. Solches sollte sich wiederfinden in den Hochschulgesetzen der erneut gebildeten Länder. Allein, wir hörten davon, der Souverän hatte wie so oft völlig daneben gewählt. So wurde es mit der radikalen Demokratisierung der Hochschulen auch von außen wieder einmal nichts.

Hochschulintern hatte die recht bald einsetzende inhaltliche Marginalisierung der aktiven Minderheiten ihre Ursache freilich nicht nur darin, daß sie die desinteressierte Mehrheit nervten. Die Marginalisierung ergab sich ebenso aus realsozialistischen Befangenheiten, denen die neuen hochschulischen Amtsinhaber offenkundig verhaftet waren. Partizipationsansprüche begegneten dominierenden Ansichten, die augenscheinlich Leninschen Argumentslinien folgten: Übergangszeiten, in denen die Frage Wer wen? noch nicht abschließend geklärt ist, benötigten vor allem eine straffe Führung. Die zu bewältigenden Anforderungen bräuchten eine harte Hand. Demokratiespiele hielten da vor allem auf. Wir könnten es uns jetzt nicht leisten, alles ewig auszudiskutieren. Wir müßten schnelle Entscheidungen treffen. Dies bedinge, daß die gewählten Funktionsträger von einem allgemeinen Vertrauen der Wähler in die Richtigkeit ihres Handelns ausgehen könnten. Mitbestimmung jeweils Betroffener und Interessierter koste zuviel Zeit und Kraft. Also: Demokratie ist ganz nett, aber nur für nette Zeiten. Die hätten wir jetzt grad mal nicht. Und weiter: Wir müßten Prioritäten setzen. Nicht so wichtiges könnten wir später regeln. Etwa diesen uns gesetzlich verdonnerten Gleichstellungszirkus. Augenblicklich stünden Dinge ganz anderer Dimension an.

Der allgegenwärtige Pragmatismus, der unablässig die nächsten Dinge zu den letzten machte, ließ der Mehrheit derartige Auffassungen einsichtig erscheinen. Zumindest immer dann, wenn es nicht gerade um die unmittelbar eigenen Interessen ging. Es verbanden sich also an den ostdeutschen Hochschulen Reflexionsdefizite mit unzulänglichen Kapazitäten der Interessenorganisierung wie mit Mängeln der Beteiligungskultur. Statt dessen überlagerten sich Anpassung und Anpassungsdruck. Der vorauseilende wie der nachholende Gehorsam wurden gleichermaßen handlungsmächtig.

Die diesbezüglich ein wenig grundsätzlicher zu stellende Frage wäre: Wie kann eigentlich in eine autoritär tradierte Gesellschaft Demokratie mit demokratischen Methoden eingeführt werden? Das mittlerweile dominieren- 
de Paradigma der Erörterung dieser Frage hatte Peter Hahne schon vor längerem (und aus gewichtigerem Anlaß: dem Zusammenschießen des russischen, zwar unsympathischen aber doch Parlaments 1993) gültig formuliert: »Nach der Niederschlagung der Opposition setzt Boris Jelzin weiter auf Demokratie. $\varkappa^{4}$ Von der Humboldt-Universität wird uns eine Szene übermittelt, die das Problem für den hiesigen Gegenstand illustriert. Sie darf als symptomatisch für die Ost-Hochschulen in den letzten Jahren gelten:

Bert Flemming, Oberarzt an der Charité, Vorsitzender der Universitätsehrenkommission und hochschulpolitischer Sprecher der SPD im Berliner Abgeordnetenhaus, *ausgerechnet der Sozialdemokrat Flemming provozierte im Juli 1991 den neuen Humboldt-Kanzler Rainer Neumann, seine bis dahin geubte Abstinenz in hochschulpolitischen Auseinandersetzungen aufzugeben. Den staunenden Mitgliedern des Akademischen Senats verkündete Oberarzt Flemming, das ... Ergänzungsgesetz zum Berliner Hochschulrecht... versage deswegen den Humboldtianern die verfassungsrechtlich garantierten Mitspracherechte in den Gremien, weil viele nicht nach rechtsstaatlichen Maßstäben in ihre Ämter gekommen seien. Das Gesetz wolle daher 'Demokratie zum Teil aussetzen' ... Der Kanzler, entgeistert, klärte Flemming darüber auf, daß es zum Wesen von Grundrechten gehöre, daß diese nicht auszusetzen sind." (Küpper 1993, 79)

Schließlich ergab sich eine bemerkenswerte Erneuerungskoalition zwischen westdeutschen Wissenschaftsgremien und ostdeutschen Administrationen einerseits und den folgsamen der OstwissenschaftlerInnen andererseits. Daß letztere meist nicht diejenigen waren, die dem Zwang eigener Ideenformulierung unterlagen, mag kein Zufall gewesen sein. Sie nahmen es erst einmal hin, daß die ostdeutschen Hochschulen bis zur Sturmreife delegitimiert wurden. Die anfängliche Rede von der »Forschungswüste《 (Zacher 1990) hatten sie zaghaft pariert mit der Schilderung ihrer eigenen Unterdrückung, die sie nicht weiter als bis zum Professor hatte kommen lassen. Euphorisch begrüßten sie dann, daß kurz darauf auch »Oasen« (Seibt 1991) in der ostdeutschen Wissenschaftslandschaft entdeckt wurden - ohne zu bemerken, daß die »Oasen« zugleich eine Referenz an die Wüsten-Metapher waren. Damit hatten sich einige von ihnen für die Mitarbeit an der Umgestaltung der Ost-Hochschulen qualifiziert.

Das an allen Hochschulen übliche wie fatale "Räsonieren der Laien《 (Teichler 1994, 169), die sich gleichwohl aufgrund der Vertretung eines wissenschaftlichen Faches auch unverzüglich als Experten für Wissenschaft und hochschulische Bildung und deren Organisation empfinden, fand sich nun um eine spezifische Facette bereichert: Glaubenssätze wurden beliebt, schließlich sei alles offenkundig. Rationale Argumentationen, etwa die Entwicklung von Begründungszusammenhängen galten als entbehrlich. Begriffliche Klärungen waren verpönt. Wissenschaftstheorie blieb unbekannt. Platter Positivismus führte zu vereinfachenden Problemdefinitionen und, daraus abgeleitet, der Formulierung unterkomplexer Pro-

4 Am 25.10.1993 in den $» H e u t e-S c h l a g z e i l e n \ll$. 
blemlösungsstrategien. Ideologieträchtige Dichotomisierungen, z.B. »systemnah - systemfern«, verbanden sich mit unablässig repetierten, trotzdem unverstandenen Slogans, die solcherart alsbald Platitüden waren: etwa 'Interdisziplinarität' oder das fortwährend dröhnend geraunte 'Hum-boldtHumboldt'. Übertüncht wurden (und werden) die gelegentlich offenkundig werdenden Defizite durch permanente Bezugnahme auf »große Traditionen « und die Revitalisierung verstaubter akademischer Formen. Das führt bei aufgeklärteren westdeutschen Gästen regelmäßig zu versteckter Heiterkeit, allerdings meist nur solange, als es sie nicht dauerhaft in den Osten verschlägt. Ereilt sie ein Ruf, wird der akademische Firlefanz nach drei Tagen spätestens locker mitgemacht: eine immer wieder zu beobachtende Ossifizierung. Das amüsanteste Beispiel hierbei ist übrigens die MartinLuther-Universität in Halle/S., die durch Senatsbeschluß die Abkürzung MLU verboten hat (Lilie 1993), wegen des u.U. mißverständlichen $\gg$ ML« darin: Sie gilt mittlerweile als der akademische Trachtenverein schlechthin, da sie regelmäßig Straßenumzüge in schwarzen Umhängen veranstaltet.

Das auch andernorts bekannte Demokratie-Expertokratie-Dilemma fand sich in Ostdeutschland verschärft durch den Wechsel des Führungspersonals. Die neuen Funktionsinhaber waren in besonderem Maße Nur-Experten, was auf ein spezifisches Transformationsdilemma verweist: Es empfahlen sich für derartige Verwendungen vomehmlich diejenigen, die in der DDR ihre Aktivitäten auf eine (bspw. fachliche) Nische beschränkt hatten und somit die politisch unbefleckte Empfängnis verkörpern konnten. Sie verschafften den ablaufenden Prozessen eine oberflächliche Legitimität und hatten im übrigen kaum Eigenständiges einzubringen, es sei denn bei der phantasievollen verfahrenstechnischen Umsetzung vorgegebener Zielgrößen.

Als wirkungsmächtiger erwiesen sich die externen Akteure, wobei Extern-Ost (vornehmlich die Landeswissenschaftsministerien) und ExternWest (diverse Gremien auf Bundes-, Länder- und Dritter Ebene, Gründungsdekane, Kommissionsmitglieder) zu unterscheiden sind. Bei westexternen Akteuren wuchs der entscheidendere Einfluß den Landeshochschulstrukturkommissionen wie den Gründungskommissionen vor Ort zu. Hier wurden politische Wünsche empfangen und Reviere gesichert. Hier wurde im Bewußtsein unmittelbarer Gestaltungswirkungen nachgedacht und formuliert.

An diesem Punkt haben mittlerweile ausgiebig dokumentierte Interpretationsdifferenzen ihre Ursache. ${ }^{6}$ Es sind dies die Differenzen zwischen

5 Das fing gleich 1989 gut an: »Wir sprechen uns für die 'Ordinarien-Universität' im Humboldt'schen Sinne aus, für die dialektische Einheit von Forschung und Lehre«, findet sich in der Stellungnahme eines Leipziger Physikers vom 13.11.1989. (Brandsch 1989, 1)

6 Die heftigste Debatte diesbezüglich hat der seinerzeitige Wissenschaftsratsvorsitzende Dieter Simon ausgelöst, vgl. Simon (1995). 
gleichsam den Architekten - da insonderheit der Wissenschaftsrat zu nennen - und den Bauleitern - Gründungsdekane, Vertreter der Fachverbände u. dgl. - des ostdeutschen Hochschulumbaus. Wo erstere funktionale Flexibilität, stilistische Vielfalt und bauliche Eleganz in den Mittelpunkt ihrer Entwürfe für die zuvor planierte Landschaft zu rücken suchten, da gingen letztere meist weit traditioneller von Überlegungen $\mathrm{zu}$ Baumaterial und Statik aus. Davon leiteten sie alles weitere ab, und als elegant galten nun vor allem einheitliche Traufhöhen. Die Architekten sahen in manchen Punkten durchaus die Chance zur systemischen Korrektur, die Bauleiter die zur Selbsterhöhung. Erstere sahen das urbane Ensemble, letztere ihre konkrete Baustelle. Die Architekten sind heute die kenntnisreichen Analytiker einer vertanen großen Gelegenheit - leider nur meist ohne den Anteil eigener Mißperzeptionen, mangelhafter Kräfteeinschätzung und der Überbewertung systeminterner Innovationskapazitäten zu reflektieren, mithin dieselben Schwierigkeiten bei schonungsloser Selbstevaluierung aufweisend, wie sie nach 1990 an den DDR-WissenschaftlerInnen kritisiert worden waren. Die Bauleiter sind heute großteils Ehrendoktoren ihrer jeweiligen Hervorbringung.

Letztere waren den wesentlichen externen Ost-Akteuren, den Landesministerien, inhaltlich und geographisch näher. Das wesentlich verschaffte ihnen die bessere Durchsetzungskraft. Dabei half, daß die Dezentralisierung - ein prioritärer Topos des Umbaus in Auswertung der DDR-Erfahrungen bei den Landesministerien endete. Die Folge war eine weitgehend direktive ministeriale Steuerung der Hochschulen in den Jahren bis 1994.

Dieserart konnte zunächst eine Struktur etabliert werden, die in den wesentlichen Punkten eine Ableitung westdeutscher Gegebenheiten ist. Was im Westen seit geraumer Zeit als »verrottet (Simon 1991) gilt, war für den Osten erst einmal der Gipfel der Erneuerung. Gradmesser des Erfolgs wurde das erreichte Maß der nachholenden Annäherung an die westdeutsche Hochschul-Moderne. ${ }^{7}$ Entstanden ist solcherart eine konventionelle

$7 \mathrm{Zu}$ diesem Struktur-, insbesondere Institutionentransfer finden sich sozialwissenschaftliche Erklärungsversuche. Vgl. etwa Eichener et al. $(1992,548)$ : Bei »aller Kritik im einzelnen« habe "praktisch keine Alternative zu diesem Vorgehen existiert... Da sich ein politisch-administratives System nicht gleichzeitig transferieren und reformieren läßt, weist die Vereinigung zwangsläufig strukturkonservative Züge auf« - eine Vorstellung, die den Transfer als unabdingbare Prämisse alles weiteren setzt. Indessen wäre es bei einer "Alternative zu diesem Vorgehen « nicht schlechterdings um reformierenden Transfer, sondern um eine bestimmte Prioritätensetzung zwischen Transfer und Reform gegangen, letztere verbunden mit der Integration definierter Elemente und insofern eines Transfers hinüber und herüber. Doch hätte das Definitionserfordernis zu bewußten Integrations- und Transferentscheidungen im Grundsätzlichen wie im Einzelfall genötigt. Die Transferperspektive muß in der Tat Reformen, als Störfaktoren des Transfers, ausschließen. Eine Reformperspektive hätte dagegen auf die Betrachtung des Transfers als 'Sachzwang' zu verzichten gehabt. 
Struktur, die noch in ihren (beschränkten) Innovationen Konventionsverstöße meidet. Die schwache Vertretung z.B. universitärer Frauenforschung in Ostdeutschland illustriert das offenkundige Unvermögen, Innovation als die Förderung des Noch-nicht-Mehrheitsfähigen zu betrachten. Wo an westdeutschen Hochschulen, wenn nicht verfestigte Überzeugungen, so doch wenigstens kosmetische Überlegungen zu einer gewissen institutionalisierten Frauenforschungsdichte führen, da finden sich in Ostdeutschland ganze drei entsprechend denominierte Professuren in den gesamten universitären Geistes- und Sozialwissenschaften (und zwei davon an der Humboldt-Universität). Die entsprechende Widerstandsfähigkeit entstand als deutsch-deutsche Bewährungsprobe, wie ein Beispiel aus der Ostberliner Theologie, die einigermaßen ost-west-gleichberechtigt umstrukturiert worden war, veranschaulichen mag. Eine Aktivistin für die Verankerung feministischer Lehr- und Forschungsinhalte erinnerte sich nach dem Ende der (nicht sehr erfolgreichen) Auseinandersetzungen einer eindrücklichen Zirkularität der innovationsbremsenden Argumentationen, die als exemplarisch gelten darf:

»Feministische Theologie als eigenes theologisches Fach entspreche nicht dem gegenwärtig sich in der Theologie artikulierenden hermeneutischen und historisch-wissenschaftichen Interesse, hieß es. Eben deshalb schien es uns zunächst allein über die Einrichtung einer Professur möglich, dauerhaft zu sichern, daß feministische Theologie bereits angemessen betrieben werden kann, bevor sich das gegenwärtige Theologieverständnis geändert hat. Doch diesem (inhaltlichen) Einwand war vorgebeugt worden: Auch die vorgegebene Sollstruktur verhindere die Einrichtung einer zusätzlichen Professur. Dann also, so unser nächster Vorstoß, in allen Bereichen die Sicherung feministischer Forschung und Lehre über Assistenzstellen. Doch nein, so wurde uns beschieden, dies würde die Wissenschaftsfreiheit der Lehrstuhlinhaber beeinträchtigen, die über die Stellenvergabe an wissenschaftliche Mitarbeiter zu entscheiden hätten - und die, wie hinzuzufügen wäre, im Normalfall dem 'gegenwärtig sich in der Theologie artikulierenden hermeneutischen und historisch-wissenschaftlichem Interesse' folgen. Darauf hätten wir nur wieder antworten können: Also, wie die Dinge liegen, benötigt die feministische Theologie unbedingt einen eigenen Lehrstuhl, um überhaupt stattfinden zu können.«(Klank 1995, 44)

Selbstredend brachte die Implantation der konventionellen West-Struktur bei allen Verwerfungen auch Schätzenswertes mit sich. Die regionale Auffächerung hochschulischer Angebote und die flächendeckende Vertretung der meisten Fächer bspw. machen institutionelle und disziplinäre Konzentrationstendenzen rückgängig, wie sie die DDR angestrebt und großteils umgesetzt hatte. In einem weiter gefaßten Betrachtungshorizont sind indessen andere Entwicklungen wesentlicher: Die Schwerpunktsetzung auf die Entwicklung von Fachhochschulen zulasten der Universitäten steht für Differenzierung, die einem ideologisch aufgeladenen Begabungsbegriff folgt. Vorgänge wie die gesetzliche Einführung sog. starker Dekane befriedigen nicht nur ein genuin ostdeutsches professorales Bedürfnis, son-

8 Wohinter im übrigen weniger böse Absichten steckten, sondern eher eine andere Systemlogik, die auf die Planungsfähigkeit des Zusammenhangs von In- und Output baute. 
dern stehen auch für Bestrebungen nach hochschulinterner Oligarchisierung. Drittmittelfixiertheit und Orientierung auf anwendungsorientierte Forschung perpetuieren einen ökonomistischen Wissenschaftsbegriff, der den ostdeutschen WissenschaftlerInnen mehrheitlich nicht erst eingebleut werden mußte.

Sind für diese Vorgänge auf der Ebene politischer Betrachtung die beiden zentralen und gegensätzlichen Deutungsachsen »Erfolgsstory« (Rüttgers 1996, 182) und »Wissenschaftskatastrophe《 (Richter/Wipperfürth o.J.) meinungsstrukturierend geworden, so herrscht auf der Ebene systematischer Beurteilung die Begriffsfigur der 'konservativen Modernisierung' vor. Renate Mayntz stellte fest, daß es zu einer konservativen Zieldefinition gekommen sei, deren Verfolgung zwar für Ostdeutschland »einen kurzfristig zu bewerkstelligenden, radikalen Wandel« bedeutete, daß für eine umfassende, d.h. darüber hinaus gehende Reformanstrengung dagegen »alle wesentlichen Voraussetzungen« gefehlt hätten: Diese Anstrengung hätte

seinen entsprechenden Reformwillen und ein Reformkonzept vorausgesetzt; bei fehlendem Konsens unter den direkt und indirekt (über ihr Widerstandspotential) an einer solchen Reform Beteiligten hätte es eines durchsetzungsfähigen dominanten Akteurs bedurft, der die Reform planen und ihre Implementation sichern konnte. Die bloße Tatsache, daß das bundesdeutsche Hochschulwesen vor der Vereinigung als eminent reformbedürftig galt, genügte nicht, da unter den wichtigsten Entscheidungsbeteiligten kein Konsens über die zentralen Ursachen der Mängel, über die Reformziele und über die zu ergreifenden Maßnahmen bestand.« (Mayntz 1994, 308f)

Ein wenig polemischer ließe sich auch formulieren: 1990 gab es in Ostdeutschland eine Wissenschaftskrise und in Westdeutschland eine Wissenschaftskrise. Beide sind unterdessen erfolgreich zusammengeführt worden. Wenn wir ein wenig Komparatistik treiben wollten, dann können wir in der Tat zu überraschenden Befunden gelangen. Etwa indem wir - einseitig, gewiß - nach Parallelen suchten zwischen den Funktionen von DDR- und gesamtdeutschem Hochschulsystem: Wo dieses die notwendigen Kader für das eine System heranzuzüchten hatte, da hat jenes die Bereitstellung der Funktionselite für das andere System zu leisten. Wo dieses Beiträge zur Meisterung der Wissenschaftlich-technischen Revolution im Dienste der sozialistischen Revolution erbringen sollte, da ist jenem ein Part in der forschungsseitigen Bewältigung des technologischen Wandels zur Sicherung des Standortes Deutschland aufgetragen. Wo dieses Innovativität vor allem in der Bombastik der Rechenschaftslegung kannte, da sorgen in jenem Verteilungskämpfe und -kompromisse wie verbeamtet-professorale Strukturen für die Verkrustung der Institutionen. Wo in diesem Mitbestimmung der Hochschulangehörigen über staatlich-einheitsparteilich gelenkte Organisationen (nicht) geregelt wurde, da läßt in jenem eine verfassungsgerichtlich abgesicherte Professorendominanz Mitbestimmung zu einem ornamentalen Ereignis werden. 
Wir könnten, so wir zur Polemik neigten, beinahe vom Versuch einer gesamtdeutschen Rekonstruktion des DDR-Hochschulwesens sprechen (unter Ausschluß lediglich der dortigen ideologischen Überformungen mit ihren methodischen und inhaltlichen Auswirkungen sowie der DDR-typischen Personalstruktur). Anwendungsorientiertheit der Forschung, drastische Eingriffsmöglichkeiten der Ministerien, Bildung als Standortfaktor, selektierter Hochschulzugang, normierte Studiengänge, sanktionsbewehrte Hochschulaufenthaltszeiten, vorrangig berufsqualifizierender Charakter des Studiums u. dgl.: Das sind Stichworte, in denen sich die seit 1990 forcierte gesamtdeutsche Debatte mit inhaltlichen Ansprüchen des DDRHochschulsysterns trifft.

Es sind Stichworte zudem, die auch die einzig wesentlichen Neuerungen in den ostdeutschen Hochschulgesetzen benennen. Was in diesen westabweichend vorkommen durfte, waren solcherart bezeichnete Verschärfungen gegenüber der westdeutschen Gemütlichkeit. Während im Westen noch heftig diskutiert wurde, schrieb es der Osten einfach in seine Gesetze. Der sächsische Wissenschaftsminister weiß auch, warum: "Der Spinner und Flenner, der Chaoten und Scharlatane gibt es in Deutschland weiß Gott mehr als genug. Deren Schar brauchen die Hochschulen nicht zu vermehren.« (Meyer 1993)

Fassen wir bis hierher zusammen: Der ostdeutsche Hochschulumbau hatte sich, im Rahmen einer vollendenden Modernisierung Ostdeutschlands, als ein Anpassungsproze $\beta$ an das normsetzende und strukturtransferierende westdeutsche Hochschulsystem vollzogen. Er war motivational hochideologisiert und von Partikularinteressen überfrachtet, in der mentalen Verwurzelung autoritär fixiert, zur demokratischen Legitimitätsfindung erneuerungspolitisch dekoriert, zur Identitätsverschaffung traditionalistischer Ornamentik verhaftet und in der juristischen Grundlegung der personellen Umgestaltung naturrechtlich orientiert. Er war gekennzeichnet durch Schwächen der Problemdefinition, in ihrem Anspruchsniveau stark differierende Zielsetzungen der verschiedenen Akteure, Delegitimierungsanstrengungen hinsichtlich der Ausgangsstruktur, asymmetrische Organisiertheit der konfligierenden Interessen, dadurch dominierenden Einfluß klientelistischer (westdeutscher) Interessenstrukturen, im weiteren durch Reflexionsschwächen zentraler ostdeutscher Akteure und auf westdeutscher Seite getragen vom Überlegenheitsempfinden des - zwar »verrotteten « aber: - westdeutschen Hochschulsystems. Schließlich war er gekennzeichnet durch einen unangemessenen Zeitrahmen, defizitäre Transformationsbewältigungskapazitäten des politischen Systems sowie Sparzwänge. Diese prozeßbestimmenden Elemente bewirkten eine Neustrukturierung, die im Vollzug bürokratisch von Steuerungshavarien und demokratisch von Legitimitätsdefiziten geprägt sowie überwiegend innovationsresistent war. In 
seinen Ergebnissen führte der Vorgang zu einem weitgehenden aber nicht vollständigen akademischen Elitenwechsel, zur Verwestlichung wie Vermännlichung des Personals und zu einer Struktur, die auch in ibren (beschränkten) Innovationen Konventionsverstöße - Innovation verstanden als Förderung des Noch-nicht-Mehrheitsfähigen - weitestgehend meidet. Der Gesamtvorgang wirkte, strukturbezogen, ost- und westdeutsche Hochschulkrisen vereinheitlichend und, handlungsbezogen, auf die fraglose Annahme der neuen Verhältnisse zurichtend. Für letzteres erfüllte der Prozeß nicht zuletzt eine hochsymbolische Funktion: Die Delegitimierung des DDR-Systems ließ sich wirksam bewerkstelligen über die symbolische Delegitimierung der alten DDR-Funktionseliten. Diese wiederum war am einfachsten an den Hochschulen zu inszenieren, da sie über nur extrem schwache Sanktionspotentiale gegenüber dem regieführenden Staat verfügen.

All das muß nicht verwundern. Es sollte aber gewußt werden. Dann läßt sich die Ostentwicklung zutreffender in den gesamtdeutschen Kontext einordnen. $\mathrm{Zu}$ »Krise und Zukunft der Universität« (lies wohl: der Hochschule) hat die ostdeutsche Hochschulentwicklung seit 1990 einen technokratisch-konservativen Beitrag geleistet. Damit unterscheidet sich der Vorgang grundsätzlich von den nach-68er Hochschulentwicklungen West, in denen sich Technokraten und Demokraten verbündet hatten - und mit denen der soeben stattgefundene Ostumbau immer einmal wieder verglichen worden ist. Die Programmierungssoftware für die Implementation Ost wird zwar für künftige westdeutsche Anwendungen nicht umstandslos einsetzbar sein, da dort deutlich besser organisierter Klientelismus das Anwendungsfeld weit komplexer gestaltet. Jedoch können die Ostentwicklungen dem gesamtdeutschen Erfolg des konservativ-technokratischen Bündnisses insofern förderlich sein, als sie die Legitimitätsdefizite des bestehenden Hochschulsystems durch Verweis auf bereits erprobte, 'besser funktionierende' Ansätze in Ostdeutschland zusätzlich verstärken.

\section{Literatur}

Brandsch, H. (1989): Vorschläge zu den notwendigen Veränderungen an der Universität, Leipzig, unveröff., zit. in: P. Pasternack, Hochschule in die Demokratie. Fallstudie (KarlMarx-)Universität Leipzig 1989-1993, Berlin 1995, 76.

Brentjes, Sonja; Pasternack, Peer (1994): Berufungsverfahren Ost: Der Spagat zwischen Selbst- und Fremdergänzung, in: hochschule ost, Vol. 3, Nr. 4, 28-43.

Eichener, Volker; Kleinfeld, Ralf; Pollack, Detlef; Schmidt, Josef; Schubert, Klaus; Voelzkow, Helmut (1992) ( $\mathrm{Hg}$. ): Organisierte Interessen in Ostdeutschland, Marburg 1992.

Klank, Irene (1995): Erfahrungen mit der Institutionalisierung feministischer Theologie in Berlin, in: hochschule ost, Vol. 4, Nr. 4, 40-45.

Küpper, Mechthild (1993): Die Humboldt-Universität. Einheitsschmerzen zwischen Selbstreform und Abwicklung, Berlin.

Lilie, Hans (1993): Senats-Beschluß, die Abkürzung »MLU« nicht mehr zu gebrauchen, in: Universitätszeitung der Martin-Luther-Universität Halle-Wittenberg, Nr. 2.

Mayntz, Renate (1994): Die Erneuerung der ostdeutschen Universitäten zwischen Selbstre- 
form und externer Intervention, in: dies. (Hg.), Aufbruch und Reform von oben. Ostdeutsche Universitäten im Transformationsprozeß, Frankfurt a.M./New York, 283-312.

Melis, Charles (1993): Was ist von dem DDR-Forschungspotential übriggeblieben?, in: H. Schramm (Hg.), Hochschule im Umbruch. Zwischenbilanz Ost, Berlin, 350-363.

Meske, Werner (1993): Die Umgestaltung des ostdeutschen Forschungssystems. Eine Zwischenbilanz, hrsg. vom Wissenschaftszentrum Berlin für Sozialforschung, Berlin.

Meyer, Hans Joachim (1993): Hans Joachim Meyer anläßlich der Einweihung der Chemnitzer Wirtschaftswissenschaftlichen Fakultät 1993, in: Deutsche Universitätszeitung, Vol. 49, Nr. 23,83 .

Pasternack, Peer (1995): Hochschule in die Demokratie. Die personelle Emeuerung an der Universität Leipzig: eine empirische Probe aufs demokratische Exempel, in: Das Hochschulwesen, Vol. 43, Nr. 3, 152-160.

Pasternack, Peer (1996): Geisteswissenschaftler heute. Das geisteswissenschaftliche Forschungspersonal in Ostdeutschland nach fünf Jahren Strukturwandel, in: Das Hochschulwesen, Vol. 44, Nr. 3, 156-161.

Richter, Edelbert/Wipperfürth, Christian (o.J.) [1992]: Wissenschaftskatastrophe. Zur Situation von Forschung und Hochschulen in den newen Ländern. hrsg. von den SPD-Abgeordneten im Europäischen Parlament, o.O. (Strasbourg).

Rüttgers, Jürgen (1996): Fünf Jahre deutsche Einheit: Die blühenden Landschaften sind überall im Kommen, in: hochschule ost, Vol. 5, Nr. 1, 182-186.

Seibt, Gustav (1991): Oasen in Sicht. Wissenschaft im Test: Die Akademie der DDR wurde evaluiert, in: Frankfurter Allgemeine, 16.7.1991, 23.

Simon, Dieter (1991): Dic Uni ist verrottet, in: Der Spiegel, Nr. 50, $52 \mathrm{f}$.

Simon, Dieter (1995): Verschleudert und verschludert, in: Die Zeit, Nr. 15, $39 \mathrm{f}$.

Teichler, Ulrich (1994): Hochschulforschung. Situation und Perspektiven, in: Das Hochschulwesen, Vol. 42, Nr. 4, 169-177.

Wissenschaftsrat (1996): Stellenausstattung der Hochschulen in den alten Ländern leicht gestiegen - Stellenabbau in den neuen Ländem, in: hochschule ost, Vol. 5, Nr. 3, 164f.

Zacher, Hans F. (1990): zit. in: Wüste. Kritik an der DDR-Wissenschaft (AP), in: Frankfurter Allgemeine, 21.6.1990, 31 . 